\section{Beihilfe zum Suizid kann eine ärztliche Handlung sein*}

P. Baumann

Gelegentliche Zusammenarbeit mit Exit bringt mir zutiefst befriedigende Erlebnisse und, selten, Stunden voller quälend unlösbarer Probleme. Exit gibt zur Herbeiführung des Freitodes Na-Pentobarbital in letaler Dosierung. Vorerst wird in mehreren Gesprächen geprüft, ob der Sterbewunsch eindeutig und zeitlich konstant ist. Dann hat ein Arzt nach Durchsicht der vorliegenden medizinischen Befunde zu entscheiden, ob diese die Ausstellung des benötigten Rezepts rechtfertigen und ob die Urteilsfähigkeit genügend intakt ist. Schon vor der ersten Kontaktaufnahme unterscheiden sich diese Menschen grundlegend von den Suizidalen, die die Ärzte in Klinik und Praxis sonst sehen: sie geben keinen Behandlungsauftrag und wenden sich rechtzeitig an das vorerst noch anonyme Sekretariat einer Sterbehilfeorganisation.

Der Zürcher Kantonsarzt Dr. U. Gabathuler hat in einem Brief an die Ärzte des Kantons die Rechtslage für die Rezeptierung von Na-Pentobarbital in letaler Dosis klar zusammengefasst. Danach ist die Rezeptierung bei urteilsfähigen Sterbenden, die das wollen, möglich. "Das Verwaltungsgericht hat aber festgehalten, es sei äusserst fraglich, Patienten mit offensichtlich nicht lebenslimitierenden Krankheiten, wie psychische Erkrankungen, als Sterbende zu bezeichnen. Bei der Ausstellung eines Rezeptes ist die Zeugnisstellung somit äusserst wichtig. Einerseits muss die zum Tode führende Erkrankung medizinisch entsprechend gut dokumentiert und überprüft worden und anderseits muss die gegebene Urteilsfähigkeit der $\mathrm{Pa}-$ tientin bzw. des Patienten in bezug auf den Todeswunsch sorgfältig schriftlich dokumentiert sein. Beide Elemente müssen gegeben sein, der Wunsch einer Patientin bzw. eines Patienten alleine, zu sterben, genügt nicht."

Damit teilen sich die Menschen, die Exit um Hilfe zum gewollten Sterben bitten, in zwei Kategorien: diejenigen, die bald sterben müssen, die körperlich Todkranken, und die, die nur sterben möchten. Bei

* Eingereicht am 15. September 2000. Auf die aktuelle politische Diskussion um den Zürcher Stadtratsbeschluss wird also nicht eingegangen.

Korrespondenz:

Dr. med. Peter Baumann

Feldeggstrasse 65

CH-8008 Zürich den ersteren ist es mir gestattet, das von Exit benötigte Rezept für eine tödliche Dosis von Na-Pentobarbital auszustellen.

Der zweiten Kategorie dagegen muss ich die Erfüllung des Wunsches verweigern, wenn nicht Exit die Toleranz der Behörden verlieren will und ich meine Praxisbewilligung. Wenn der Suizidwunsch dringend und einfühlbar ist und mich menschlich richtig dünkt - dieser Ermessensspielraum wirkt oft als Willkür, muss aber dem Arzt erhalten bleiben -, kann diese Beschränkung hart sein.

Schwierige Grenzfälle sind z.B. nicht beherrschbare Schmerzen bei Vorliegen eines nicht heilbaren, nicht bald tödlichen, progredienten körperlichen Leidens, etwa bei polymorbiden, lebensmüden sehr alten Menschen.

Das Beisein eines Arztes beim Suizid verlangt Exit zur Vermeidung von Zweifeln in jenen Fällen, wo wegen Schluckstörung oder wegen Brechneigung das Sterbemittel über eine Infusion (die der Sterbende selber in Gang setzt) verabreicht wird. Diese Sterbebegleitungen, bei denen der Sterbeprozess in Minuten abläuft, sind ärztlich-menschlich immer zutiefst beglückende Momente, wo der Kranke seine Ambivalenzen endgültig hinter sich gelassen hat, die emotionalen Reste mit den Nächsten bereinigt sind und der Kranke gelöst und dankbar in den Tod geht, manchmal fröhlich, manchmal traurig, ernst, liebend, immer ruhig, immer ohne Auflehnung, immer dankbar für das, was ihm das Leben gegeben hat und dafür, dass ihm der hässliche, böse Tod erspart wird. Wenn ich in den Sekunden, bevor ich die Kanüle in den Körper steche, in mich hineinhorche, mein Gewissen prüfe und auf die Stimmen der ethischen Instanz, auch der vor-rationalen, religiösen, und des Heilers in mir höre, vernehme ich stets deren Zustimmung, uneingeschränkt, und ich weiss: diese Beihilfe ist eine ärztliche Tätigkeit.

Und sie erscheint mir sogar eindeutig als heilende Tätigkeit. An die Stelle des Sich-Auslieferns an die Krankheit tritt der gewollte, gewählte Tod, das Übernehmen der Verantwortung für ein Lebensende nach sorgfältigem Erwägen und Festlegen des nach menschlichem Ermessen richtigsten Zeitpunkts. Ich möchte das einen gesunden Tod nennen, ein reifes Akzeptieren und Mitgestalten des Unabwendbaren, den Durchbruch zur Eindeutigkeit, gut, im Einklang mit dem höheren Selbst, in anderer Sprache "gottgefällig».

Wie diese Sterbestunden erlebe ich auch die Abklärungssitzung, bei der ich zu entscheiden habe, ob Pentothal oder nicht, als therapeutisch. Hier geht es um die nochmalige Klärung der Motivation und um die Offenlegung irrationaler Ängste: Darf man das? Gegen den Willen der vermuteten öffentlichen Meinung, des strafsüchtigen Gottes, oder der Mutter, die für diese Todsünde, nämlich ihre Zustimmung zu dieser Todsünde, in die Hölle zu müssen fürchtet? Stets achte ich darauf, dem Petenten (er ist ja Gesuchsteller und erlebt sich nicht als mein Patient) gegenüber möglichst genau und aufrichtig $\mathrm{zu}$ begründen, warum ich so oder so entscheide, wie die ärztlich- 
rationale Instanz urteilt und wie meine unmittelbaren Antwortgefühle sind, ob ich vorbehaltlos zustimme, überzeugt ablehne oder contre cœur einen durch Exit begleiteten Suizid verweigern muss. Wenn ich zustimme und der Kranke sich im Wissen verabschiedet, dass damit nun alles vorbereitet ist, sehe ich oft an seinen nassen Augen und höre in bewegten Worten, dass ich ihn aus der diesseitigen Hölle des unfreiwilligen Weiterlebenmüssens hinausbegnadigt habe in einen würdigen, weil möglichst selbstbestimmten, letzten Lebensabschnitt. Viele erleben durch diese Angstentlastung eine wesentliche Besserung ihres Zustands, und viele machen von der jetzt jederzeit offenen Sterbemöglichkeit erst nach weiteren Wochen oder Monaten oder gar nicht Gebrauch.

Nun zu den Menschen, die sich mit ihrem Sterbewunsch über Exit an mich wenden, ohne körperlich todkrank zu sein. Wenn wir alle diese Menschen, wie es heute üblich ist, als (psychisch) krank bezeichnen, haben wir schon entschieden für das morbozentrische Menschenverständnis und über unser Recht, als Aussenstehende über gesund und krank zu urteilen, für moralische Behandlungspflicht des "Patienten" und dafür, dass unsere Prognose ("grundsätzlich nie sicher unheilbar») derjenigen des Betroffenen ("hoffnungslos») überzuordnen ist. Auch dafür, dass diese oft mit Grund sehr vertrauensgestörten Menschen weiterhin das nötige Vorschussvertrauen in unsere ärztliche Kunst haben sollen - deren Grenzen uns ja an sich deutlich sind. Wir wissen schliesslich um die Effektivität weiterer Therapien bei «notorischen Therapieversagern». Weiterhin Antidepressiva ut aliquid fiat, oder gar FFE ut aliquid fieri videatur, damit es aussieht, als gehe etwas?

Die Menschen, die mir unter der sachfremden Vorausetikette "psychisch krank" zur Untersuchung und Beurteilung geschickt wurden, sind bei nosographischem Denken den Charakterneurosen zuzuordnen. Sie sehen den Beginn ihrer Suizidalität immer in der Kindheit oder Adoleszenz. Der gemeine alte Lebensekel ist in ihren Gesichtern zu sehen und aus ihren Geschichten zu hören, und eine bewusste oder latente riesige Enttäuschungswut. Oft sind sie schon ein Stück des Weges in die Verwahrlosung gegangen mit Aussteuerung, IV-Rente, FFE's, Sucht, und möchten auf diesem Weg, der sie sicher nicht wieder freilassen wird, nicht weitergehen.

Ich erwähnte schon, dass Spitalärzte grundlegend anders geartete Suizidale sehen als Exit-Mitarbeiter. Die einen Suizidalen, Patienten in einem Spital, haben sich in totale Abhängigkeit begeben, wo sie über den FFE jederzeit zum Weiterleben gezwungen werden könnten. Die anderen, Bittsteller oder Forderer, sind handlungsfähig und gehen den Weg über eine Sterbehilfeorganisation, bereit, mehreren Menschen gegenüber ihre Absicht zu vertreten, ihre Not zu zeigen und die Gründe darzulegen, derentwegen sie auf eine Wende dieser Not nicht mehr hoffen mögen.
Diese Wesensunterschiede machen wohl die Mediendiskussionen zwischen Exponenten der Sterbehilfe und der Suizidprophylaxe oft so missverständlich und ergebnisarm.

Wenn ich die Prognose betrachte, die persönlichen Ressourcen und die Psychopharmako- und Psychotherapien, die der Petent immer schon hinter sich hat, bin ich immer wieder überrascht, wie sehr meine Beurteilung mit der seinen übereinstimmt, wie blässlich daneben jeder professionelle Optimismus würde, wie leer alle wichtigen Ressourcenfelder sind, Urvertrauen, Glaubens-, Liebes- und Freudefähigkeit, Sexualleben, Partnerschaft, Beruf, Geld, die Fähigkeit zum Erregen von Sympathie, zum Wahrnehmen selbst banaler Freundlichkeiten - vielleicht auch zum Hoffen an sich, vor jeder Begründung.

Meine unmittelbaren Antwortgefühle waren in allen Fällen eindeutig, ob dafür oder dagegen. Ich vermute, dass das in der Sache selbst begründet ist, dass die Frage mit ihrer Absolutheit eine klare Gefühlsantwort hervorruft.

Wenn ich mir vorstellte, ich dürfte bei diesen nicht körperlich Todkranken eine tödliche Schlafmitteldosis verschreiben, spürte ich zu meinem Erstaunen auch Gefühle von Missbrauchtwerden, und zwar nicht so sehr durch mein Gegenüber, als vielmehr durch das System, das diese Entscheidung an uns Ärzte delegieren würde. Dadurch wurde ich gewahr, dass die Entscheidung hier nicht dem Arzt obliegen soll, sondern allein dem Sterbewilligen. Ich finde also unsere rechtlichen Regelungen und deren Handhabung durch die Behörden richtig und wünsche mir keine Änderung der strafrechtlichen Tötungsverbote - mit einer Ausnahme: den polymorbiden, lebensmüden Alten möchte ich den leichten Weg über das Schlafmittel ermöglichen dürfen.

Welche Möglichkeiten ärztlicher Suizidbeihilfe haben wir dort, wo die letale Barbituratdosis verboten ist?

- Eine im Grund positive, nicht dissuasive Einstellung ist erlaubt. Wir dürfen für die Meinung werben, dass Suizid eine ebenso gute, rechte Sterbeart ist wie alle anderen üblichen, und sicher nicht weniger "natürlich" als diese. Wenn der Wunsch eines Menschen, zu sterben, als überlegte Äusserung eines mündigen Erwachsenen aufgenommen wird, dem gegenüber wir offen sind, ist das Wichtigste verändert.

- Wir dürfen Wissen publizieren: wie quallos, leicht und gut Sterben gestaltet werden kann. Wie unverboten es ist, rechtlich wie biblisch. Was es braucht, um vor dem Sterben den Frieden mit sich zu finden. Dass Sterbefasten (nicht «Nahrungsdeprivation»!) eine palliativmedizinische Kultur und Technik werden kann. Dass der Wunsch, von einem Menschen bei der Vorbereitung und Durchführung des Suizids begleitet zu werden, recht ist und nicht etwa feige. 
Ich möchte, dass wir einen Verein Suizidhilfe Schweiz initiieren mit einer Sektion Ärzte, der

- der Selbstverantwortlichkeit des Menschen für sein Leben- und Sterbenwollen absolut verpflichtet ist;

- Gefässe für unvoreingenommenen Meinungsaustausch schafft;

- Gefässe schafft für die Reflexion unserer (rationalen oder irrationalen?) Angst vor der Feme der Kollegenschaft;

- Wissen verbreitet über sichere Suizidformen;

- hilft bei der Überprüfung der Entscheidung und bei der psychischen und sachlichen Vorbereitung;

- vielleicht bei der Durchführung begleitet.
Vermutlich - und das wäre bei genauerem Hinsehen nicht mehr paradox - hätte ein solcher Verein einen suizidprophylaktischen Effekt: fünf Sechstel der Anrufer einer Suizidberatungsstelle in Holland waren zwei Jahre später noch am Leben. Und ausserdem bin ich sicher, dass eine solche Entwicklung des öffentlichen Bewusstseins sehr lebensfreundlich wirken könnte: wenn Suizid normal ist, ist das Leben um einen Grad freiwilliger, und wir können uns soviel freier "das Leben nehmen» im Sinne des carpe diem, pflücken, anpacken. 